\title{
Gedanken Experiment for Fluctuation of Mass of a Graviton, Based on the Trace of GR Stress Energy Tensor-Pre Planckian Conditions that Lead to Gaining of Graviton Mass, and Planckian Conditions That Lead to Graviton Mass Shrinking to $10^{-62}$ Grams
}

\author{
Andrew Walcott Beckwith \\ Physics Department, Chongqing University Huxi Campus, Chongqing, China \\ Email:Rwill9955b@gmail.com, abeckwith@uh.edu
}

Received 29 October 2015; accepted 26 December 2015; published 29 December 2015

Copyright (c) 2016 by author and Scientific Research Publishing Inc.

This work is licensed under the Creative Commons Attribution International License (CC BY).

http://creativecommons.org/licenses/by/4.0/

\section{(c) (1) Open Access}

\begin{abstract}
We will be looking at the energy of a graviton, based upon the Stress energy tensor, and from there ascertaining how fluctuations in early universe conditions impact the mass of a graviton. Physically the mass of the graviton would be shrinking right after Planck time and presumably it would be going to its equilibrium value of about $10^{-62}$ grams, for its present day value. It, graviton mass, would increase up to the Plank time of about $10^{-44}$ seconds. Note that the result that graviton mass shrinks to $10^{-62}$ grams for its present day value works only for relic gravitons.
\end{abstract}

Keywords

Heavy Gravity, Plank Time, Stress Energy Tensor

1. Introduction, Setting up for Calculation of Using the Results of Initial Energy as Due to $\delta t \Delta E=\frac{\hbar}{\delta g_{t t}} \equiv \frac{\hbar}{a^{2}(t) \cdot \phi}$ and Comparing It to a More General Energy

Expression Given Below

Start off with looking at from [1], a generalized energy expression with momentum also obeying, if $m$ is for How to cite this paper: Beckwith, A.W. (2016) Gedanken Experiment for Fluctuation of Mass of a Graviton, Based on the Trace of GR Stress Energy Tensor-Pre Planckian Conditions that Lead to Gaining of Graviton Mass, and Planckian Conditions That Lead to Graviton Mass Shrinking to $10^{-62}$ Grams. Journal of High Energy Physics, Gravitation and Cosmology, 2, $19-24$. http://dx.doi.org/10.4236/jhepgc.2016.21002 
graviton mass. Begin with from [1].

$$
p=\frac{h}{\lambda}
$$

$$
\begin{aligned}
& p=\frac{h}{\lambda} \\
& \& E=p c+\frac{m^{2} c^{2}}{2 E} \\
& \Rightarrow E \sim \frac{h c}{2 \lambda}\left(1 \pm \sqrt{1+\frac{4 m^{2} c^{4}}{\left(\frac{h c}{\lambda}\right)^{2}}}\right) \sim \frac{h c}{2 \lambda}\left(2+\frac{2 m^{2} \lambda^{2} c^{4}}{(h \cdot c)^{2}}\right), \text { Or } \sim \frac{h c}{2 \lambda}\left(-\frac{2 m^{2} \lambda^{2} c^{4}}{(h \cdot c)^{2}}\right)
\end{aligned}
$$

Next, from Giovannini [2], if T is the trace of the Stress-Energy tensor, we have that

$$
\begin{gathered}
\left(m=m_{\text {graviton }}\right)^{2}=-\frac{\kappa}{6} T \\
E_{\text {graviton }} \sim \frac{h c}{2 \lambda}\left(2-\frac{\kappa T \lambda^{2} c^{4}}{3(h \cdot c)^{2}}\right), \text { Or } \sim \frac{h c}{2 \lambda}\left(-\frac{\kappa T \lambda^{2} c^{4}}{3(h \cdot c)^{2}}\right)
\end{gathered}
$$

If so, then, the fluctuation of energy would be represented, if $\lambda_{\text {graviton }}=\frac{2 \pi v(\text { velocity })_{\text {graviton }}}{\omega_{\text {graviton }}}$ and we have [3]

$$
\left(\frac{v_{\text {graviton }}}{c}\right)^{2}=1-\frac{m_{\text {graviton }}^{2} c^{4}}{E_{\text {graviton }}^{2}}
$$

Then if we go to look at what [1] $\lambda_{\text {graviton }}=\frac{2 \pi v(\text { velocity })_{\text {graviton }}}{\omega_{\text {graviton }}}$ then is saying, the above is then rendered as

$$
\begin{aligned}
& \left(E_{\text {graviton }}\right)^{2} \sim\left[\frac{h c}{2 \lambda}\left(2-\frac{\kappa T \lambda^{2} c^{4}}{3(h \cdot c)^{2}}\right)\right]^{2}, \text { Or } \sim\left[\frac{h c}{2 \lambda}\left(-\frac{\kappa T \lambda^{2} c^{4}}{3(h \cdot c)^{2}}\right)\right]^{2} \sim \frac{m_{\text {graviton }}^{2}(\text { rest-mass }) c^{4}}{\left(1-\left[v_{\text {graviton }}(\text { velocity }) / c\right]^{2}\right)} \\
& \Leftrightarrow m_{\text {graviton }}^{2}(\text { rest-mass }) \sim \frac{\left(1-\left[v_{\text {graviton }}(\text { velocity }) / c\right]^{2}\right) c^{2}}{72 \pi^{2} \hbar^{2}} \\
& \quad \times\left[\kappa \cdot T\left(=\text { trace }_{u v}\right) \cdot\left(\lambda_{\text {graviton }}=\frac{2 \pi v(\text { velocity })_{\text {graviton }}}{\omega_{\text {graviton }}}\right)\right]^{2}
\end{aligned}
$$

Note that the presence of the mass reduces the speed of gravitons with respect to the speed of light. As has stressed repeatedly by Dr. Corda and his collaborators. Corda and collaborators have stated that this massive graviton mass leads to a situation for which the graviton generates a longitudinal component of strain in the arms of a interferometric detector. This point is made abundantly clear in clarified in the papers of Prof. Corda and collaborators, i.e. see references [4]-[9]. This will be of crucial importance in the concluding remarks of this document. Next. Let us consider what happens if there is a fluctuation in Graviton mass. 


\section{Utilizing Equation (6) in Terms of the Initial Fluctuation of the Graviton Mass}

From [1] [10]-[12], use

$$
\begin{aligned}
& T\left(=\text { traceT }_{u v}\right) \approx \Delta T_{t t} \sim \Delta \rho \sim \frac{\Delta E}{V^{(3)}} \\
& \frac{\Delta E}{V^{(3)}}=\frac{\hbar}{\delta t \cdot \delta g_{t t} \cdot V^{(3)}} \equiv \frac{\hbar}{\delta t \cdot V^{(3)} \cdot a^{2}(t) \cdot \phi} \\
& \Leftrightarrow \Delta m_{\text {graviton }}^{2}(\text { rest-mass }) \sim \frac{\left(1-\left[v(\text { velocity })_{\text {graviton }} / c\right]^{2}\right) c^{2}}{72 \pi^{2} \hbar} \\
& \quad \times\left[\frac{\kappa}{\delta t \cdot V^{(3)} \cdot a^{2}(t) \cdot \phi} \cdot\left(\frac{2 \pi v(\text { velocity })_{\text {graviton }}}{\Delta \omega_{\text {graviton }}}\right)\right]^{2}
\end{aligned}
$$

\section{Identifying Change in $\Delta \omega_{\text {graviton }}$ : This Is the Input into Equation (7), Assuming $v(\text { velocity })_{\text {graviton }} \sim 0.98 \% c($ light - speed $)$}

We follow what to expect from $\Delta T_{t t} \sim \Delta \rho \sim \frac{\Delta E}{V^{(3)}}$ as given in [1] [2] for

$$
\delta t \Delta E=\frac{\hbar}{\delta g_{t t}} \equiv \frac{\hbar}{a^{2}(t) \cdot \phi}
$$

as a way to quantify energy density when we have what is coming from Weinberg [12] on initial energy density and then from there to say something about initial time step and also potential energy as given Padmanbhan [1]. Doing so will isolate out values of the Potential energy, as in [12] which will then be compared to [1]'s potential energy value, which in turn gets a value of time, which we will set by first considering the following evolution equation. From [12],

$$
\ddot{\phi}+3 H \dot{\phi}+\partial_{\phi} V(\phi)=0
$$

Then, look at $V(\phi)$ from [12] as having the value of, if $\mathrm{M}$ is related to mass, with $\alpha$ a variable parameter, which can be negative, with then a smallest value of $\alpha=-4$, and a frequent value of $\alpha=-2$ as in the case of chaotic inflation. Here in general

$$
V(\phi)=M^{4+\alpha} / \phi^{\alpha}
$$

The parameter $\alpha=-2$ in the chase of chaotic inflation, i.e. one of the simplest models, whereas it can be positive up to $\alpha=2$ in other models. The results are though that with $-4 \leq \alpha \leq 2$

So, then the $\phi$ is given by [12]

$$
\phi=\left(\frac{\alpha \cdot(\alpha+2)^{2} \cdot M^{4+\alpha} \cdot t^{2}}{6+\alpha}\right)^{\frac{1}{\alpha+2}}
$$

And also look at Padmanabhan's generalized inflation potential [12], of comparing Equation (2) with Equation (12) below

$$
V=\frac{3 H^{2}}{8 \pi G} \cdot\left(1+\frac{\dot{H}}{3 H^{2}}\right)
$$


We have the Hubble parameter, if before Planck time, during Plank time $\dot{H}= \pm \delta H$

$$
\begin{aligned}
& H=H_{\text {initial }} \mathrm{e}^{ \pm \delta t} \Leftrightarrow \dot{H}= \pm \delta H, \\
& \dot{H}=+\delta H \text { if Before Planckian time } \\
& \dot{H}=-\delta H \text { if Planckian time zone }
\end{aligned}
$$

Then, we could get the following variance in time, $\tilde{t} \sim \Delta t$

$$
\begin{aligned}
& \phi=\left(\frac{\alpha \cdot(\alpha+2)^{2} \cdot M^{4+\alpha} \cdot t^{2}}{6+\alpha}\right)^{\frac{1}{\alpha+2}} \approx\left(\frac{8 \pi G M^{4+\alpha}}{( \pm \delta-3 H) \cdot H}\right)^{\frac{1}{\alpha}} \\
& \Leftrightarrow \tilde{t}=\left(\frac{M^{\left(\frac{4+\alpha}{2 \alpha}\right) \cdot(2-\alpha)}}{H_{\text {initial }} \exp ( \pm \delta \cdot t)}\right) \cdot\left(\frac{6+\alpha}{\alpha \cdot(2+\alpha)^{2}}\right)^{\frac{1}{\alpha+2}} \cdot\left(\frac{8 \pi G}{\left( \pm \delta-3 \cdot H_{\text {initial }} \exp ( \pm \delta \cdot t)\right)}\right)^{\frac{1}{\alpha}}
\end{aligned}
$$

\section{Finding How to Use this Value of $\tilde{t} \sim \Delta t$ in Order to Estimate a Relic GW Frequency}

If so, then, up to a point, in the Pre Plankian regime of space time, according to the signs on Equation (13) and Equation (14) and [10] [11] for the change in $\delta t \Delta E=\frac{\hbar}{\delta g_{t t}} \equiv \frac{\hbar}{a^{2}(t) \cdot \phi}$ Set then, in early universe conditions,

let us set, if we are considering gravitons, that we will set, say that the expression below would be for pre Planckian times, with $t<10^{-44}$ seconds. The upshot would be that there would be a GW frequency, in many cases, as a result of pre Planckian physics of greater than or equal $10^{32} \mathrm{~Hz}$, which would be red shifted down to about $10^{10} \mathrm{~Hz}$, i.e. a 22 order of magnitude drop, in the present era. This is assuming $a^{2}$ (initial) $10^{-110}$, as well as we are assuming $N \sim 10^{37}$, as seen in [10] [11]

$$
\begin{aligned}
& \delta t \Delta E=\frac{\hbar}{\delta g_{t t}} \equiv \frac{\hbar}{a^{2}(t) \cdot \phi}=\delta t \cdot N_{\text {gravitons }} \cdot \hbar \cdot \omega_{\text {graviton }} \\
& \Leftrightarrow \omega_{\text {graviton-initial }} \approx \frac{1}{N_{\text {gravitons }} \cdot a^{2}(t) \cdot \phi} \\
& \approx \frac{1}{N_{\text {gravitons }} \cdot a^{2}(t)} \cdot \frac{\left(\frac{6+\alpha}{\alpha \cdot(2+\alpha)^{2}}\right)^{\frac{-1}{\alpha+2}} \cdot\left(\frac{8 \pi G}{\left( \pm \delta-3 \cdot H_{\text {initial }} \exp ( \pm \delta \cdot t)\right)}\right)^{\frac{-1}{\alpha}}}{\left(\frac{\left.M^{\left(\frac{4+\alpha}{2 \alpha}\right) \cdot(2-\alpha)}\right)}{H_{\text {initial }} \exp ( \pm \delta \cdot t)}\right)}
\end{aligned}
$$

The $M$ as given in this would correspond to the Mass value of the universe, which is roughly $3 \times 10^{55} \mathrm{~g}$ (where $g$ is for grams) [13].

\section{Conclusion: Putting Equation (15) into Equation (7). What It Says, Physically}

Note that time in Equation (14) remains finite but very small, as it came out less than 10 to the minus 44 power seconds, less than Planck time, with the parameter $\alpha$ usually less than 2. Time, in Equation (14) as estimate is actually negative, unless we have that we chose in Equation (14) the Pre Planckian option, which is saying that likely Planck time may not be the earliest sub division of time as we know it. This last point above will be important in our future research. As well as entropy production models due to discussions in [14]-[18] in terms of 
entropy generation in the Pre Planckian era. The entropy values will influence the $\mathrm{N}$ used in Equation (15) above. After this is set, for Equation (15) we put Equation (15) into Equation (7) and thereby obtain

$$
\begin{aligned}
& \Delta m_{\text {graviton }}^{2}(\text { rest-mass }) \sim \frac{\left(1-\left[v(\text { velocity })_{\text {graviton }} / c\right]^{2}\right) c^{2}}{72 \pi^{2} \hbar} \\
& \times\left[\frac{\kappa}{\delta t \cdot V^{(3)}} \cdot \frac{\left(2 \pi v(\text { velocity })_{\text {graviton }}\right)}{\left(\frac{M^{4+\alpha}}{H_{\text {initial }} \exp ( \pm \delta \cdot t)}\right)^{\frac{1}{\alpha}}}\right] \times\left(\frac{1}{N_{\text {gravitons }}} \cdot \frac{\left(\frac{6+\alpha}{\alpha \cdot(2+\alpha)^{2}}\right)^{\frac{-1}{\alpha+2}}}{\left(\frac{\left.M^{\left(\frac{4+\alpha}{2 \alpha}\right) \cdot(2-\alpha)}\right)}{H_{\text {initial }} \exp ( \pm \delta \cdot t)}\right)}\right)^{-2}
\end{aligned}
$$

The first term of Equation (16) roughly cancels with the number of gravitons, which approximately leaves

$$
\Delta m_{\text {graviton }}^{2}(\text { rest-mass }) \sim \frac{c^{2}}{72 \pi^{2} \hbar} \times\left[\frac{\kappa}{\delta t \cdot V^{(3)}} \cdot \frac{\left(2 \pi v(\text { velocity })_{\text {graviton }}\right)}{\left(\frac{M^{4+\alpha}}{H_{\text {initial }} \exp ( \pm \delta \cdot t)}\right)^{\frac{1}{\alpha}}}\right] \times\left(\frac{\left(\frac{6+\alpha}{\alpha \cdot(2+\alpha)^{2}}\right)^{\frac{-1}{\alpha+2}}}{\left(\frac{M^{\left(\frac{4+\alpha}{2 \alpha}\right) \cdot(2-\alpha)}}{H_{\text {initial }} \exp ( \pm \delta \cdot t)}\right)}\right)^{-2}
$$

The change in graviton mass is not so much affected by $N$, entropy count, as this is partly neutralized by the near speed of light conditions, for massive gravitons. What is left though is the variation in total mass, $M$ is divided by $H_{\text {initial }} \exp ( \pm \delta \cdot t)$, which expands during the Pre Planckian space-time regime, and which shrinks right after Planckian time is breached, in the Planckian era (the Universe begins a massive deceleration. The term $\alpha$ would usually be expected to be less than 2. With $-4 \leq \alpha \leq 2$, and $\alpha=-2$ chosen in the case of the very simple chaotic inflationary model.

Physically what this is saying is that the mass of the graviton would be shrinking right after Planck time and presumably it would be going to its equilibrium value of about $10^{-62}$ grams [19], for its present day value. It, graviton mass, would increase up to the Plank time of about $10^{-44}$ seconds, i.e. the graviton will shrink to $10^{-62}$ grams in the onset of inflation right after $10^{-44}$ seconds, for a stable value of rest graviton mass up to the present day. We also, again state the critical importance of [4]-[9] as far as the physics, of the following statement that a massive graviton generates a longitudinal component of strain in the arms of an interferometric detector and that the simplest model to investigate as far as relic conditions would be the chaotic inflationary model chosen if $\alpha=-2$.

\section{Acknowledgements}

This work is supported in part by National Nature Science Foundation of China grant No. 11375279.

\section{References}

[1] Padmanabhan, T. (2006) An Invitation to Astrophysics. World Scientific, Co. Pte., Singapore.

[2] Giovannini, M. (2008) A primer on the Physics of the Cosmic Microwave Background. World Scientific, Singapore.

[3] Will, C. (2006) The Confrontation between General Relativity and Experiment. Living Reviews in Relativity, 9. http://relativity.livingreviews.org/Articles/lrr-2006-3/

[4] Crowell, L. and Corda, C. (2014) f(R) Gravity, Relic Coherent Gravitons and Optical Chaos. Galaxies, 2, $160-188$. http://www.mdpi.com/2075-4434/2/1/160

[5] Corda, C. (2010) Massive Relic Gravitational Waves from f(R) Theories of Gravity: Production and Potential Detec- 
tion. The European Physical Journal C, 65, 257-267. http://arxiv.org/abs/1007.4077

[6] Corda, C. (2009) Interferometric Detection of Gravitational Waves: The Definitive Test for General Relativity. International Journal of Modern Physics D, 18, 2275-2282. http://arxiv.org/abs/0905.2502 http://dx.doi.org/10.1142/S0218271809015904

[7] Capozziello, S., Corda, C. and De Laurentis, M.F. (2008) Massive Gravitational Waves from f(R) Theories of Gravity: Potential Detection with LISA. Physics Letters B, 669, 255-259.

http://www.sciencedirect.com/science/journal/03702693/669 http://dx.doi.org/10.1016/j.physletb.2008.10.001

[8] Corda, C. and Mosquera Cuesta, H.J. (2009) A Spherically Symmetric and Stationary Universe from a Weak Modification of General Relativity. Europhysics Letters Association • EPL (Europhysics Letters), 86, No. 2. http://iopscience.iop.org/article/10.1209/0295-5075/86/20004/meta;jsessionid=4325631A1108AF986616AA0570ED2 0D0.c1.iopscience.cld.iop.org

[9] Corda, C. (2007) A Longitudinal Component in Massive Gravitational Waves Arising from a Bimetric Theory of Gravity. Astroparticle Physics, 28, 247-250. http://arxiv.org/abs/0811.0985 http://dx.doi.org/10.1016/j.astropartphys.2007.05.009

[10] Beckwith, A. (2015) Gedanken Experiment for Degree of Flatness, or Lack of, in Early Universe Conditions. Accepted for publication in JHEPGC October 22. http://vixra.org/pdf/1510.0108v4.pdf

[11] Beckwith, A. (n.d.) Gedanken experiment for Refining the Unruh Metric Tensor Uncertainty Principle via Schwartzshield Geometry and Planckian Space-Time with Initial Non Zero Entropy and Applying the RiemannianPenrose Inequality and the Initial Kinetic Energy for a Lower Bound to the Graviton. Under review for publication in the Ukrainian Journal of Physics. http://vixra.org/abs/1509.0173

[12] Weinberg, S. (2008) Cosmology. Oxford University Press, Oxford, UK.

[13] Valev, D. (2010) Estimations of Total Mass and Energy of the Universe. http://arxiv.org/pdf/1004.1035v1.pdf

[14] Ha, Y.K. (2014) An Underlying Theory for Gravity. Proceedings of the 7th international conference on Gravity and Cosmology (ICGC 2011), Journal of Physics: Conference Series, 484, 012061. http://iopscience.iop.org/1742-6596/484/1/012061/pdf/1742-6596_484_1_012061.pdf http://dx.doi.org/10.1088/1742-6596/484/1/012061

[15] Ng, Y.J. (2007) Holographic Foam, Dark Energy and Infinite Statistics. Physics Letters B, 657, 10-14. http://dx.doi.org/10.1016/j.physletb.2007.09.052

[16] Ng, Y.J. (2008) Spacetime Foam: From Entropy and Holography to Infinite Statistics and Nonlocality. Entropy, 10, 441-461. http://dx.doi.org/10.3390/e10040441

[17] Kolb, E. and Turner, M. (1990) The Early Universe. Frontiers in Physics, Vol. 69, Chicago, Illinois, USA,

[18] Mukhanov, Y. (2005) Physical Foundations of Cosmology. Cambridge University Press, Cambridge, UK. http://dx.doi.org/10.1017/CBO9780511790553

[19] Goldhaber, A.S. and Nieto, M.M. (2010) Photon and Graviton Mass Limits. Reviews of Modern Physics, 83, 939-979. http://arxiv.org/abs/0809.1003 